\title{
Daily sodium intake as a function of time of measurement and formalin injection volume and concentration
}

\author{
JOHN J. PORTER and HELMUT RELINGER \\ University of Wisconsin-Milwaukee, Milwaukee, Wis. 53201
}

The effects of alternate-day injections of $.25, .75$, or $1.0 \mathrm{ml}$ of either $1.5 \%$ or $.5 \%$ formalin subcutaneously upon saline consumption of a $.33-\mathrm{M}$ solution were investigated over 8 days. Generally, percentage formalin differences produced the same saline intake, except when measured 8 rather than $24 \mathrm{~h}$ after injection when the slower recruitment of sodium need with a $.5 \%$ solution was evident. The $1.0 \mathrm{ml}$ dosage produced significantly greater saline ingestion across other experimental conditions, while the two lesser formalin volumes did hot produce a significant difference in intake. The main effects of formalin injection were on Day 1, with some less impressive effects occurring after Injections 2 and 3.

Past research (cf. Wolf \& Steinbaum, 1965) has shown that subcutaneous formalin injections produce both a loss of sodium from general circulation and increased sodium appetite, as evidenced by rate of saline $(\mathrm{NaCl})$ intake in the rat. The method by which formalin injections produced sodium deprivation has been studied in detail (cf. Stricker, 1966; Wolf \& Steinbaum, 1965). These studies have shown that sodium deficiency reaches its maximum approximately $8 \mathrm{~h}$ after injection and that recovery was apparently complete $16 \mathrm{~h}$ later. In a study of the effect of volume of formalin injected upon saline intake, Handal (1965a) found that very small volumes of formalin $(.15 \mathrm{ml})$ were sufficient to potentiate saline intake and that the appetitive effect of formalin on sodium intake approached asymptote at $.75 \mathrm{ml}$ of $1.5 \%$ formalin. This concentration has been used by the vast majority of investigators of formalin-induced sodium need.

The studies noted above indicated that a strong sodium need could be rapidly produced by injection of a relatively small volume of formalin. Though these results tend to point out the promising nature of formalin injections as a source of drive, there seems to be no data on formalin-produced sodium need over more than a $48 \cdot h$ period. Since many investigations of motivation and changes in motivation (cf. McDonald, Porter, \& Madison, 1970) require the presence of a drive state over several days, the need for a study of formalin-produced sodium need over several days was clear. Though Wolf and Steinbaum stated that formalin injections were well tolerated and could be repeated a number of times in the same animal, they did note that the injections should be spaced a few days apart. The present authors were unable to find published data on the effects of successive formalin injections on sodium need.

In the present study the effects of different volumes of repeated formalin injections were studied. The injections were administered on alternate days over an 8-day period. Though Smith, Stricker, \& Morrison (1969) and Handal (1965a) have shown that injections of $1.5 \%$ formalin will elicit the intake of saline concentrations $(.5 \mathrm{M})$ that are aversive to the normal rat (Handal, 1965b), pilot work in our laboratory has shown that repeated injections of $1.5 \%$ formalin produced considerable skin irritation in the rat at the injection site. This was the case even if injections were administered every second day and if different sites were chosen. The present study used groups injected with either $.5 \%$ or $1.5 \%$ formalin in order to see if the weaker solution could elicit consistent sodium intake without skin irritation. Finally, measurements of sodium intake were taken either 8 and $32 \mathrm{~h}$ after injection or 24 and $48 \mathrm{~h}$ after injection in order to see if weaker solutions of formalin resulted in slower recruitment of the sodium need.

\section{SUBJECTS AND DESIGN}

Forty eight Holtzman albino rats, approximately 180 days old and from 360 to $500 \mathrm{~g}$, were used. Ss were assigned to three different classes and assigned equally to each of the experimental groups. Twelve Ss were assigned to the four cells of a factorial design, with $1.5 \%$ or $.5 \%$ formalin and 8-32- or 24-48-h measurement periods as factors. Each group of 12 Ss was then subdivided into four cells according to the volume of their injections.

\section{PROCEDURE}

For the first 2 days, all Ss were water deprived for $24 \mathrm{~h}$ and then received $1 / 2 \mathrm{~h}$ exposure to the test cages, which contained a water bottle filled with distilled water and a 100-ml graduated drinking tube filled with $.33 \mathrm{M} \mathrm{NaCl}$. The nozzles of both drinking tubes and water bottles entered the front of the test cages approximately $7 \mathrm{~cm}$ apart. Ss presumably learned the respective test cage positions of the water and saline solution during the 2 days of pretraining.

After their second exposure to the test cages, all Ss were returned to their home cages and given distilled water and a modified sodium-deficient diet (Nutritional Biochemicals Co.) ad lib. On Day 3 the $1.5 \%$ groups (A and $B$ ) were subcutaneously injected with .25 , .75 , or $1.00 \mathrm{ml}$ of formalin or a sham injection of $.75 \mathrm{ml}$ of $.15 \mathrm{M}$ saline. The .5\% groups (C and $D$ ) were then injected in the same manner as above, with the same amount. Eight hours after injection, Groups $\mathrm{A}$ and $\mathrm{C}$ were placed in test cages for a $1 / 2-h$ period and then returned to their home cages. Saline consumption was recorded for each $S$ and then recorded again $32 \mathrm{~h}$ after injection. Twenty-four and $48 \mathrm{~h}$ after injection, Groups $B$ and D were tested in the same fashion as Groups $A$ and C. After $48 \mathrm{~h}$ had elapsed, all Ss in all groups were again injected with their respective concentrations and amounts of formalin. This procedure was repeated until each $S$ had received a total of four injections over a period of 8 days.

\section{RESULTS}

The present results were analyzed in two sections. The first analysis treated saline consumption over the first 2 days, theperiod following the first injection, in order to provide a direct comparison with past research. The second analysis covered saline consumption over the entire 8 days of the experiment and was concerned with changes in saline intake patterns following repeated injections. Statistical comparisons of experimental and control group performance are not included because the control Ss drank at a uniformly lower rate than the formalin injected Ss throughout the majority of the study.

On Days 1 and 2 , a 2 by 2 by 2 by 3 analysis of variance over days (D), percentage formalin (PF), hours of measurement (HM), and amount injected (AI) variables was conducted. Several significant main effects occurred: $\mathrm{HM}, \mathrm{F}(1,24)=6.76$, $\mathrm{p}<.025 ; \mathrm{AI}, \mathrm{F}(2,24)=9.68, \mathrm{p}<.01$; and $D, F(1,42)=155.02, p<.01$. However, in each of these cases, the interpretation of main effects was tied to at least one significant interaction, necessitating an analysis of simple effects in order to pinpoint the source of each effect.

The significant $\mathrm{HM}$ effect was tied to a significant interaction between 


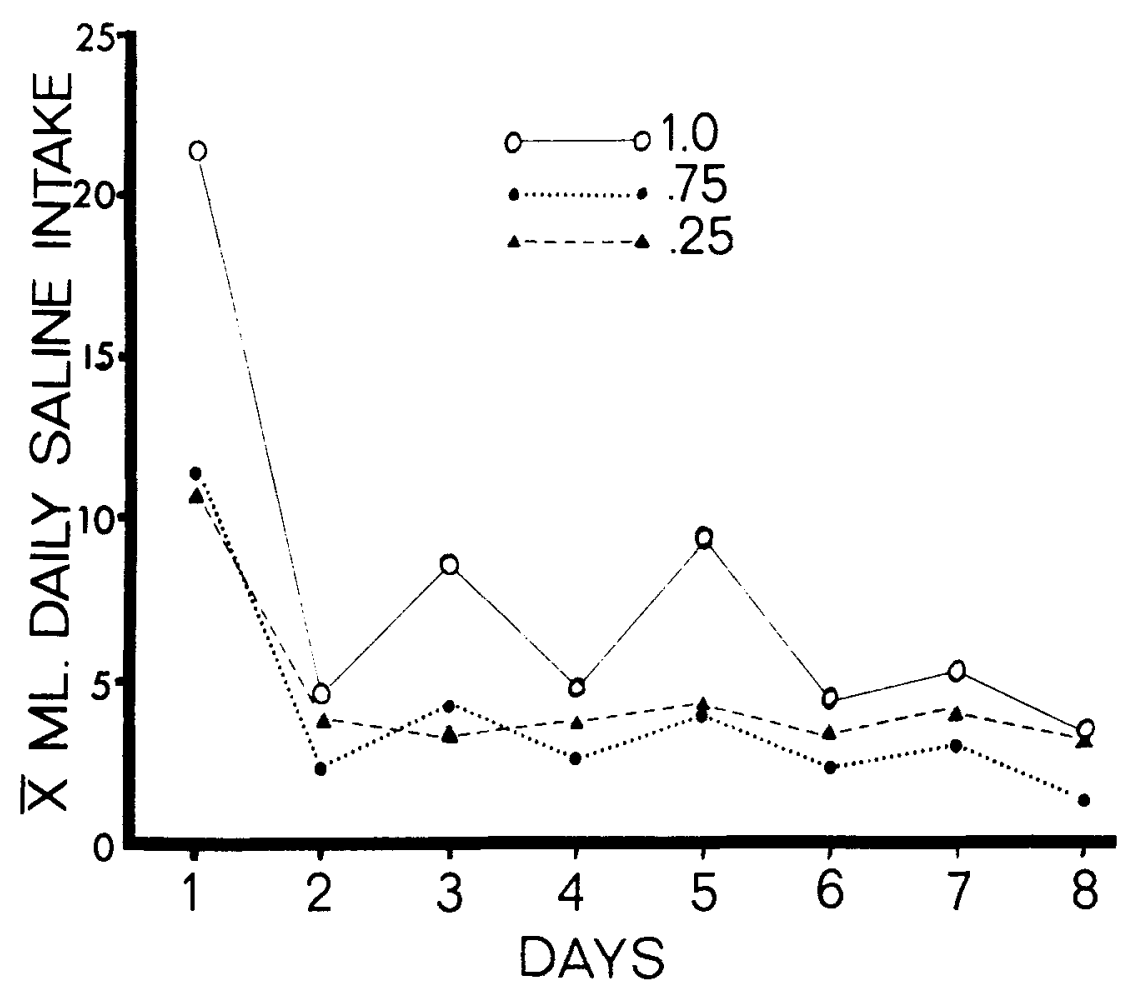

Fig. 1. Mean saline intake on Days 1.8 as a function of amount injected. The points on the figure are collapsed across hour of measurement and percentage formalin variables.

$\mathrm{HM}$ and $\mathrm{PF}, \mathrm{F}(1,24)=7.83, \mathrm{p}<.01$. As was clear from Fig. 1, a simple effects analysis demonstrated that the HM effect was meaningful only for the $.5 \%$ groups, $\mathrm{F}(1,24)=14.57, \mathrm{p}<.01$. For the $.5 \%$ groups, the lesser saline intake after 8 as compared to $24 \mathrm{~h}$ indicated slower recruitment of the sodium need than with $1.5 \%$ solutions. Further examination of Fig. 1 clearly indicated that the source of the significant $\mathrm{Al}$ effect was the apparent superiority in saline intake of the 1.0-m l groups. A Tukey multiple-comparisons test (Kirk, 1968) confirmed this observation $(p<.01)$, and indicated that the saline intake of the .25- and .75-ml groups did not differ reliably.

Figure 1 presents the data for Day 1 alone since there were virtually no noticeable effects of any of the variables on Day 2. This was confirmed by examining the source of the interaction between $A I$ and $D$, $\mathrm{F}(2,42)=11.67, \mathrm{p}<.01$, where simple effects analyses indicated that the AI effect was due to differences in saline intake on Day $1, F(2,48)=$ $21.92, \mathrm{p}<.01$. Finally, the overall $\mathrm{D}$ effect and the interaction between $D$ and $H M$, and D and AI, reemphasized that the primary effect of formalin injection occurred on Day 1.

Figure 2 displays the saline intake over Days 1-8 as a function of AI. In Fig. 2, the picture of saline intake is collapsed over $\mathrm{PF}$ and $\mathrm{HM}$ variables, since, as indicated below, these variables did not exert a significant effect upon saline intake after Day 1. There was a significant decrease in saline consumption over days, $F(7,168)=43.46, p<.01$. Tukey multiple-comparison tests indicated that saline consumption on Day 1 was significantly greater than on all other days $(p<.01)$. Saline consumption on Postinjection Days 3 and 5 was significantly greater than on remaining days $(p<.01)$, which did not differ significantly. As was clear from Fig. 2, the overall AI effect, $\mathrm{F}(2,24)=7.27$, $\mathrm{p}<.01$, was due to the greater saline consumption of the $1.0-\mathrm{ml}$ Ss as compared to the .75- and .25-ml Ss, an observation confirmed by multiple-comparison tests $(p<.01)$. The significant $\mathrm{D}$ by $\mathrm{AI}$ interaction, $F(14,168)=3.94, p<.01$, was due to the effect of AI on Days 1, 3, and 5 $(p<.01)$, the only days where the AI variable produced an apparent effect. Again, multiple comparisons over days indicated that the AI effects were due to the superior intake rate of the $1.0-\mathrm{ml} \mathrm{Ss}(\mathrm{p}<.01)$.

No effects of PF were significant in this analysis, and the only effects of HM occurred in an interaction with $D$, $F(7,168)=7.17, p<.01$, which was due almost exclusively to the significant HM effect on Day 1 , $F(1,192)=25.22, p<.01$. DISCUSSION

The absence of significant differences between the saline intake of the $.5 \%$ and $1.5 \%$ formalin groups throughout this study indicated the same degree of sodium deficiency. The slower recruitment of sodium need with a $.5 \%$ solution was the only $\mathrm{PF}$ effect that was significant. Thus, $1.5 \%$ formalin produced its maximum saline intake rate $8 \mathrm{~h}$ after injection, in agreement with Stricker (1966) and other studies, while .5\% formalin took longer than $8 \mathrm{~h}$ but less than $24 \mathrm{~h}$ to produce the same degree of saline intake. Thus when Ss were first tested $24 \mathrm{~h}$ after formalin injection, there was no significant difference in saline intake.

In the present study it was clear that the injection of $1.0 \mathrm{ml}$ of formalin led to significantly greater saline consumption than either .75 or $.25 \mathrm{ml}$. Clearly, this disagrees with Handal's (1965a) results, where the appetitive effect of formalin was asymptotic at $.75 \mathrm{ml}$. To the extent that rate of saline intake is an index of sodium deficiency, our results clearly indicated that $1.0 \mathrm{ml}$ of formalin produced a much greater degree of sodium deficiency. Thus, the possibility exists that even greater dosages may produce a greater sodium need.

The discrepancy in dosage effects and saline intake between this study and Handal's may possibly be explained by the fact that we used $.33 \mathrm{M}$ saline concentration, while $\mathrm{Handal}$ (1965 a) used .5 M concentration. Jalowiec \& Stricker (1970) found that much less .5 M saline was ingested than was isotonic $(.15 \mathrm{M})$ saline under the same formalin-produced sodium need. However, physiplogical indices indicated that the higher sodium concentration generally restored

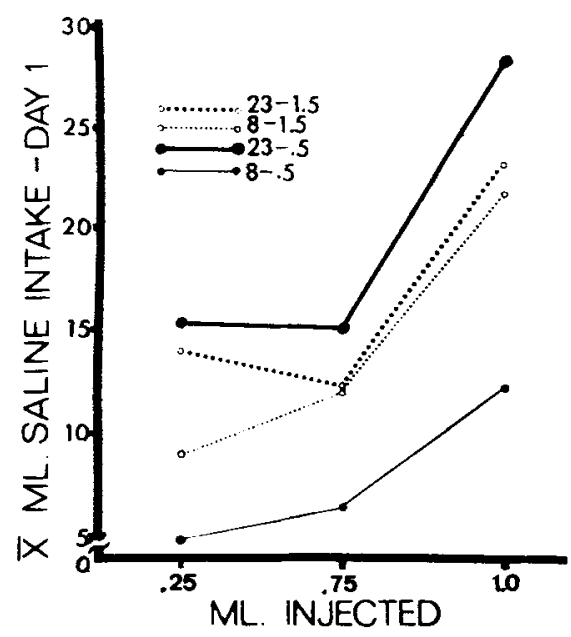

Fig. 2. Mean saline intake on Day 1 as a function of amount injected, hour of measurement, and percentage formalin injected. 
sodium balance as rapidly as $.15 \mathrm{M}$ saline, despite the smaller intake. These results would imply that in order to restore sodium balance, our $.33 \mathrm{M}$ saline solution would have required the ingestion of more saline solution for a given degree of sodium need than would Handal's .5 M solution. Thus, if amount of saline is to serve as an index of sodium need, the amount of saline ingested after various amounts of formalin injected may reach asymptote quickly with more concentrated saline reinforcement.

The decline in saline intake on the day following injections seemed clearly a result of the fact that $1.5 \%$ formalin produced maximum sodium deficiency $8 \mathrm{~h}$ after injection, whereas $.5 \%$ formalin took somewhere near $24 \mathrm{~h}$ to achieve the same effect. Thus, after the Ss were exposed to saline for their first test either 8 or $24 \mathrm{~h}$ after injection, there would be little if any sodium deficiency at the second test 32 or 48 h after injection.

A most important part of this study was the test of the effects of multiple formalin injections on Ss' behavior. Injections following the first did not produce the same degree of saline intake as the first injection. The effect of successive injections decreased, and after the fourth injection there were no significant differences in saline intake between experimental and control Ss $(p>.10)$. In light of the extensive local damage to the cellular and capillary membranes at the injection sites, produced by formalin injections, repeated injections may cause such extensive damage that a clear case of sodium deficiency is no longer evident. While Wolf \& Steinbaum (1965) noted that multiple injections could be used, it would appear that more than a few days need intervene between successive injections.

Clearly, more extensive work needs to be done with low concentrations of formalin when multiple injections are used. The results of Handal (1965a) using a $.5 \mathrm{M}$ saline solution, the present results with a $.33 \mathrm{M}$ solution, and an examination of the implications of saline concentration on rate of intake and other variables by Jalowiec \& Stricker (1970) would indicate that even lower concentrations of saline should be used in future multiple-injection studies intending to also use low formalin concentration. Thus, the use of weaker formalin concentrations should avoid the extensive external skin damage found in 12 of our 18
$1.5 \%$ concentration Ss, but found in only 2 of our $18.5 \%$ concentration Ss, while using a weaker saline solution, perhaps isotonic, should give us a more sensitive measure of sodium need produced with low concentrations of formalin.

\section{REFERENCES}

HANDAL, P J Formalin-induced sodium appetite: Dose-response relationships. Psychonomic Science, 1965a, 3, 511-512.

HANDAL. P. J. Immediate acceptance of sodium salts by sodium deficient rats. Psychonomic Science, 1965b, 3, 315-316.

JALOWIEC, J. E., \& STRICKER, E. M Restoration of body fluid balance following acute sodium deficiency in rats. Journal of Comparative \& Physiological Psychology, 1970, 70,94-102.

KIRK, R. E. Experimental design procedures for the behavioral sciences. Belmont, Calif: Brooks/Cole, 1968.

MCDONALD, S. G., PORTER, J. J., \& MADISON, H. L. Changes following asy mp totic instrumental performance as a function of shifts in drive. Psychonomic Science, 1970, 19, 33-34.

SMITH, D. F., STRICKER, E. M., \& MORRISON, G. R. $\mathrm{NaCl}$ solution acceptability by sodium-deficient rats. Physiology \& Behavior, 1969, 4, 239-243.

STRICKER E M Extracellular fluid volume and thirst. American Journal of Physiology, 1966, 211, 232-238.

WOLF, G., \& STEINBAUM, E. A. Sodium appetite elicited by subcutaneous formalin: Mechanisms of action. Journal of Comparative \& Physiological Psychology, 1965, 59, 335-339. 